\title{
Effect of a prebiotic mixture on intestinal comfort and general wellbeing in health
}

\author{
Oliver Goetze $^{1}$, Heiko Fruehauf ${ }^{1}$, Daniel Pohl ${ }^{1}$, Marianna Giarrè ${ }^{2}$, Florence Rochat ${ }^{2}$, Kurt Ornstein ${ }^{2}$, \\ Dieter Menne $^{3}$, Michael Fried ${ }^{1}$ and Miriam Thumshirn ${ }^{1,4 *}$ \\ ${ }^{1}$ Division of Gastroenterology and Hepatology, University Hospital Zürich, Zürich, Switzerland \\ ${ }^{2}$ Nutrition and Health Department, Nestlé Research Centre, Vers-chez-les-Blanc, Switzerland \\ ${ }^{3}$ Menne Biomed Consulting, Tübingen, Germany \\ ${ }^{4}$ St Claraspital, Kleinriehenstrasse 30, Basel, CH-4013, Switzerland
}

(Received 26 June 2007 - Revised 3 January 2008 - Accepted 8 February 2008 - First published online 1 April 2008)

Specific carbohydrates, i.e. prebiotics such as fructo-oligosaccharide (FOS), are not digested in the small intestine but fermented in the colon. Besides beneficial health effects of an enhanced bifidobacteria population, intestinal gas production resulting from fermentation can induce abdominal symptoms. Partial replacement with slowly fermented acacia gum may attenuate side effects. The aim was to compare the effects of FOS with those of a prebiotic mixture (50\% FOS and $50 \%$ acacia gum; BLEND) and a rapidly absorbed carbohydrate (maltodextrin) on general intestinal wellbeing, abdominal comfort and anorectal sensory function. Twenty volunteers (eight male and twelve female; age 20-37 years) completed this double-blind, randomised study with two cycles of a 2-week run-in phase ( $10 \mathrm{~g}$ maltodextrin) followed by $5 \mathrm{weeks}$ of $10 \mathrm{~g}$ FOS or BLEND once daily, separated by a 4-week wash-out interval. Abdominal symptoms and general wellbeing were documented by telephone interview or Internet twice weekly. Rectal sensations were assessed by a visual analogue scale during a rectal barostat test after FOS and BLEND treatment. Both FOS and BLEND induced more side effects than maltodextrin. Belching was more pronounced under FOS compared with $\operatorname{BLEND}(P=0.09$ for females; $P=0.01$ for males), and for self-reported general wellbeing strong sex differences were reported $(P=0.002)$. Urgency scores during rectal barostat were higher with FOS than BLEND $(P=0 \cdot 01)$. Faced with a growing range of supplemented food products, consumers may benefit from prebiotic mixtures which cause fewer abdominal side effects. Sex differences must be taken in consideration when food supplements are used.

Prebiotics: Fructo-oligosaccharides: Acacia gum: Visceral perception: Gut comfort

Prebiotics have been defined as non-digestible food compounds that selectively stimulate the intestinal flora and metabolic activity after colonic fermentation. The human colon is believed to be the predominant site of action and represents the most heavily populated intestinal region with up to $10^{12}$ colony-forming units $/ \mathrm{ml}^{(1)}$. Lactobacilli and bifidobacteria are considered indicator organisms of prebiotic stimulation; in terms of gut health, bifidobacteria are currently considered to be the most significant organisms ${ }^{(2)}$. Dietary supplements of a prebiotic such as fructo-oligosaccharides (FOS) increase the content and proportion of bifidobacteria ${ }^{(2-5)}$, which exert a positive influence on gut health. Proliferation of these bacteria allows a more predominant role for the intestinal flora as they have been shown to decrease the quantity of potentially hazardous bacteria by their metabolites and protect from intestinal infections ${ }^{(6,7)}$. Previous studies have documented positive effects on absorption of nutrients and minerals ${ }^{(8)}$, stimulation of the immune system and synthesis of vitamins ${ }^{(9)}$. Moreover, prevention of constipation ${ }^{(10)}$, colon cancer $^{(11)}$ and improve- ment of blood sugar and lipid profile ${ }^{(12)}$ are also attributed to prebiotics.

Prebiotics are also employed as fat-replacers and to improve food texture. For both health and technical reasons food products are increasingly supplemented with prebiotics. In Europe, a large range of prebiotic-supplemented food products is already available. The most commonly used prebiotics in Europe are FOS. The range of supplemented products includes (powdered) milk and fermented milk products, cereals and clinical supplements for pregnant women. FOS are naturally found in a variety of vegetables such as asparagus, leeks, artichokes, onions and garlic and have been classified as a food ingredient in the European Union, Canada and Japan ('generally recognised as safe' status in the USA) and are an acceptable food additive in Australia and New Zealand ${ }^{(13)}$. By-products of bacterial fermentation of prebiotics are gases including $\mathrm{H}_{2}, \mathrm{CO}_{2}, \mathrm{H}_{2} \mathrm{~S}$ and $\mathrm{CH}_{4}$, which may cause intestinal discomfort, i.e. flatulence, bloating and abdominal pain ${ }^{(14,15)}$. Recently, the prebiotic properties of acacia gum (Fibregum ${ }^{\circledR}$ (Colloïdes Naturels International

Abbreviations: BLEND, prebiotic mixture of fructo-oligosaccharide and acacia gum; FOS, fructo-oligosaccharide; GLMM, generalised linear mixed model;

VAS, visual analogue scale.

* Corresponding author: Dr Miriam Thumshirn, fax +41616858457, email MIRIAM.THUMSHIRN@claraspital.ch 
CNI, Rouen, France), a natural soluble fibre derived from acacia gum) have been described and a synergy for the bifidogenicity has been observed with the combination of FOS and acacia gum $^{(16)}$. Due to the growing range of supplemented products, consumers are exposed to increasing amounts of prebiotics and may ingest daily doses above the threshold for induction of side effects. In vitro fermentation time for acacia gum is significantly longer than that for FOS and studies have suggested a more favourable abdominal side-effect profile ${ }^{(17)}$.

Replacement of a proportion of FOS by acacia gum may thus attenuate the side effects of prebiotics with the additional advantage of a synergistic effect on the growth of intestinal bifidobacteria. Hence, the objectives of the present study were primarily to compare changes in intestinal comfort and secondarily to compare visceral sensitivity during rectal barostat distension following long-term ingestion of a single prebiotic (FOS), a mixture of FOS and acacia gum (BLEND) or a rapidly fermented carbohydrate (maltodextrin) in healthy volunteers. In addition, independent of treatment phases, determinants of wellbeing were analysed.

\section{Methods}

\section{Subjects and study design}

A total of eight male and twelve female healthy volunteers completed this controlled, double-blind, cross-over, randomised trial. They were recruited from the University Hospital and Universities in Zürich by in-house advertisement. Candidates with anal pathology, previous gastrointestinal surgery, pregnancy, bowel dysfunction and drug abuse were excluded from the study. Only candidates with normal findings and ingesting a similar habitual diet, as assessed by a dietary questionnaire at the screening visit, were included in the study. During a screening visit before acceptance for the study the candidates underwent haematology, blood chemistry, urine measurements, and a complete physical examination. Women of childbearing potential were required to have a negative urine pregnancy test. With the exception of birth control pills and hormone replacement therapy no regular medication was allowed during the study. Approval of the study was granted by the Ethical Committee of the University Hospital Zürich (EK-926) and all participants gave their written informed consent.

The study lasted a total of 18 weeks and was composed of five testing periods:

(1) a first 2-week run-in period during which the volunteers ingested maltodextrin, and answered a diary and a questionnaire;

(2) a 5-week ingestion period of the first product (FOS or BLEND, diary, questionnaire);

(3) a 4-week wash-out period (no product intake, diary or questionnaire);

(4) a second 2-week run-in period (maltodextrin, diary, questionnaire);

(5) a 5-week ingestion period of the second product (BLEND or FOS, diary, questionnaire).

The volunteers had to visit the study centre (visit 1, visit 2) at the end of both 5-week treatment periods. The subjects came in after a $6 \mathrm{~h}$ fasting period for stool specimen collection and barostat testing of anorectal sensory function and rectal compliance.

\section{Products}

The products used were as follows:

(1) FOS: food-grade Raftilose ${ }^{\circledR}$ P95, produced by partial hydrolysis of chicory root inulin, containing oligofructose, fructose, glucose and sucrose (ORAFTI, Tienen, Belgium).

(2) BLEND: 1:1 mixture of FOS and acacia gum, a foodgrade purified alimentary fibre derived from acacia gum (Fibregum ${ }^{\circledR}$ ) consisting of branched arabino-galactans polymers, as described previously ${ }^{(17)}$.

(3) Maltodextrin, an easily digested, neutral, slightly sweettasting carbohydrate obtained by hydrolysis of natural maize starch (Glucidex; Roquette, Lestrem, France). The starches are cooked and than acid and/or enzyme treated to break them down into smaller polymers.

\section{Doses and packaging}

The subjects were asked to ingest daily, except for the wash-out period, one sachet containing $10 \mathrm{~g}$ product $2 \mathrm{~h}$ postprandially in a drink, for example, tea, coffee, mineral water, soft drink or fruit juice. All products were packed by Nestlé (Konolfingen, Switzerland). Volunteers, support stuff and investigators were blinded regarding the ingested product. The run-in periods were not blinded since maltodextrin is sweet and easily recognised as compared with FOS or BLEND.

The subjects received the first set containing the products, questionnaires and diary for the first two periods at the screening visit (visit 0) and the second set for the last two periods at visit 1 .

\section{Questionnaire}

During the run-in and product intake periods, the volunteers answered twice weekly to a questionnaire by cellular phone or Internet. The volunteers were contacted by an automated telemedical patient data-capturing system (Medcontrol AG, 6314 Unterägeri, Switzerland). The volunteers entered information on gastrointestinal complaints and symptoms of general wellbeing into the data-capturing system. The questionnaire inquired about the occurrence and intensity (none (0), slight (1), moderate (2), strong (3)) of:

(1) pain at stomach or abdomen during last $3 \mathrm{~d}$ ('pain');

(2) feeling bloated during last $3 \mathrm{~d}$ ('bloat');

(3) being disturbed by passing wind ('wind');

(4) being disturbed by belching or burping ('belch');

(5) being disturbed by frequent bowel movements during last $3 \mathrm{~d}$ ('DiaFreq');

(6) being disturbed by urgent need to defecate ('DiaUrg');

(7) being disturbed by constipation ('Obstip');

(8) being bothered by frequent rumbling noise from stomach or gut ('borborygmi');

(9) being adversely affected by nausea ('nausea');

(10) having suffered from heart burn or acid reflux ('reflux');

(11) being negatively influenced by ingestion of the products ('general'); 
(12) being forced to reduce daily activity, i.e. job, university, household ('work').

In the figures, the sequence of the questionnaire items is reordered by the frequency of reported symptoms, with 'wind' as most frequent first, and 'reflux' as least frequent last. For quality control, a daily written diary for corresponding evaluation of bloating, pain and nausea on a four-point grading scale (none, slight, moderate, strong) and additional evaluation of stool consistency (hard, normal, unformed, watery), frequency of defecations as well as the time of product intake was filled in by the volunteers. Since corresponding questionnaire and diary outcomes correlated well in preliminary analyses only the questionnaire data are reported.

\section{Rectal barostat}

The barostat procedure was performed in a left lateral $15^{\circ}$ Trendelenburg position (the body is laid flat on the back (supine position) with the feet higher than the head). A $10 \mathrm{~cm}$-long polyethylene balloon was inserted into the rectum such that the proximal end was $5 \mathrm{~cm}$ inside the anal verge. Minimal distending pressure was determined by $1 \mathrm{mmHg} / \mathrm{min}$ stepwise rectal expansion until changes in respiratory excursion were apparent (at about $30 \mathrm{ml}$ balloon volume). Then a conditioning distension was performed, inflating the balloon by $2 \mathrm{mmHg}$ increments every $30 \mathrm{~s}$ in the range of $0-40 \mathrm{mmHg}$ to avoid changes in compliance and sensation in subsequent inflations.

Rectal compliance was assessed twice by $2 \mathrm{mmHg}$ stepwise increments of intra-balloon pressure beginning at $0 \mathrm{mmHg}$ pressure up to $40 \mathrm{mmHg}$ or until subjects reported pain. During the last $10 \mathrm{~s}$ of each pressure increase, the volunteers were asked to score their perception of lower abdominal sensation using a six-point graphic-rating scale ranging from 'no feeling' to 'painful'. After a $15 \mathrm{~min}$ break, phasic rectal distensions at 12, 24, 36 and $42 \mathrm{mmHg}$ pressure above minimal distending pressure were performed in randomised order. During the last $15 \mathrm{~s}$ of each random distension, subjects were asked to rate their abdominal symptoms, i.e. the urgency to defecate ('urgency'), bloating ('wind'), discomfort ('discomfort') and pain ('pain') using a $100 \mathrm{~mm}$ visual analogue scale (VAS) with 'none' at 0 and 'worst ever' at 100 . The equilibration interval between distensions was $2 \mathrm{~min}$ at minimal distending pressure. The method of measuring anorectal sensation and compliance was described in detail in previous studies of our laboratory ${ }^{(18,19)}$.

\section{Stool microflora}

Freshly passed faecal samples were placed into cryotubes and frozen in liquid $\mathrm{N}_{2}$ and kept at $-70^{\circ} \mathrm{C}$ until analysis at the Nestlé Research Centre using fluorescent in situ hybridisation. The probe used in the study was Bif164, specific for Bifidobacterium $^{(5)}$

\section{Data analysis and statistics}

The comparison of the questionnaire between FOS and BLEND was the primary outcome of the present study. Secondary outcomes were:
(1) comparison of questionnaire scores between treatment phase (FOS or BLEND) and run-in;

(2) intra-subject correlation of intestinal symptoms with general and work wellbeing to identify the dominant physiological correlate of intestinal comfort;

(3) comparison of visceral sensitivity under FOS and BLEND, as measured by VAS scores during rectal distension;

(4) composition of the faecal microflora under FOS and BLEND.

For the primary endpoint, questionnaire outcomes were dichotomised into 0 and 1 , where 0 means 'no symptoms reported'. The binary data were analysed with a generalised linear mixed model (GLMM), logistic link, and quasi-binomial family. Subject was the random term, sex and treatment (FOS or BLEND) and their interaction term were fixed terms; the subject's run-in means pooled over both phases were used as a covariable. The GLMM model is considered robust against unbalanced sex distributions. Results were reported as odds and OR with CI. All CI and $P$ values for the questionnaire analysis were computed as contrasts of the GLMM analysis. No adjustments for multiple testing were applied to $P$ values and CI. For questionnaire item 10 ('reflux'), which was reported by four subjects in only twenty out of 582 calls, the GLMM procedure did not converge, and therefore was removed from primary endpoint analysis. For questionnaire item 12 ('work'), the fitted probability for the male subgroup is close to zero, which can result in a high false-alarm rate; this item was also analysed by the Markov-Chain Monte-Carlo (MCMC) GLMM procedure to check for robustness.

Symptoms in the run-in phase were not significantly different from each other, and were pooled per subject. For comparison against the run-in phase as secondary outcome, a GLMM analysis was done without sex breakdown to avoid convergence problems when sex was used as a factor.

The influence of intestinal symptoms on general wellbeing ('general') and on being forced to reduce daily activity ('work') were analysed by multiple regression analysis and best predictors were reported as adjusted squared correlation coefficients.

Rectal functional parameters and threshold pressures as assessed by rectal barostat were analysed by exact Wilcoxon matched pair tests. VAS scores were transformed by an offset logarithmic transformation $(\log (\mathrm{VAS}+5))$ in order to stabilise variances and expressed as CI of VAS-equivalent balloon stimulation pressures. The slopes of VAS and graphic ratings, computed with pressure as the independent variable, served as a measure of rectal sensitivity. Slopes were determined separately for each sensation (wind, urgency, pain, discomfort), and for the sensation sum score by a linear mixed model, with subject as a random variable and treatment as fixed. For analysis of continuous data, a linear mixed model was used, using power weighting if required based on inspection of residual distribution. The logarithms of the total stool bacteria count and the bifidobacteria count were analysed by paired $t$ tests.

Data were considered significant at a level of $\alpha \leq 0 \cdot 05$. All statistics were evaluated and plotted using the open-source computing environment ' $\mathrm{R}$ ' (version 1.9.0; R Foundation for Statistical Computing, Vienna, Austria). Mixed models were 
estimated with package nlme under $\mathrm{R}^{(20)}$ and function glmmPQL in MASS ${ }^{(21)}$; the MCMC model was computed with DPpackage ${ }^{(22)}$.

\section{Results}

Eight male and twelve female healthy volunteers (median age 28 (range 20-37) years) of normal BMI (median 22.5 (range $20 \cdot 1-25 \cdot 3) \mathrm{kg} / \mathrm{m}^{2}$ ) and weight (median 67 (range 53-84) $\mathrm{kg}$ ) completed the study without any complications or adverse events. Valid questionnaire responses were obtained in 154 of 160 possible responses of the run-in phase $(96 \%)$ and in 426 of 440 possible responses $(97 \%)$ of the treatment phases, indicating very high compliance rates.

\section{Fructo-oligosaccharide v. prebiotic mixture of fructo-} oligosaccharide and acacia gum

The most frequently reported symptoms for both FOS and BLEND were 'bloating' and 'wind', with an odds over 1, corresponding to more than $50 \%$ of the calls (Fig. 1). The most prominent difference between FOS and BLEND was found for item 11 ('general wellbeing') of the male group, which was larger under FOS treatment (Fig. 2 (a)) with an OR of 9.6

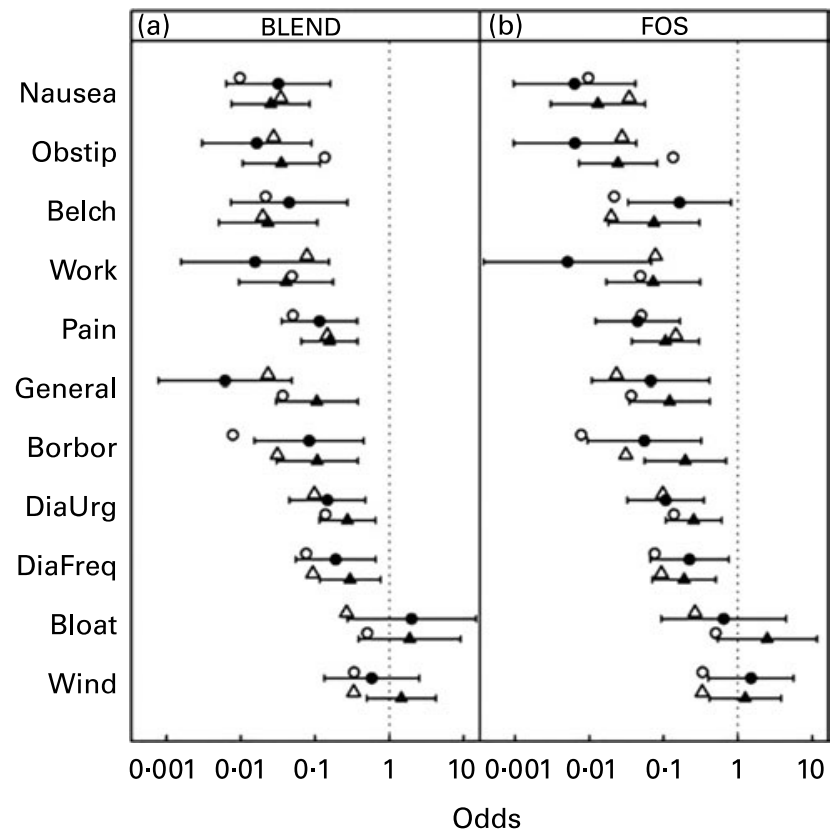

Fig. 1. Odds of reported 'symptoms present' to 'symptoms absent' for oncedaily ingestion for 5 weeks of a $10 \mathrm{~g}$ prebiotic mixture of food-grade fructooligosaccharide (FOS; Raftilose ${ }^{\circledR}$ P95) and acacia gum (1:1; BLEND) (a) and for once-daily ingestion for 5 weeks of $10 \mathrm{~g}$ FOS (b). Horizontal bars represent $95 \% \mathrm{Cl}$ from generalised linear mixed model analysis of questionnaire data. $\mathrm{Cl}$ are between-subjects and it is not valid to visualise within-subject tests against run-in. Odds are on a logarithmic scale; the dotted vertical line shows the value of $50 \%$ reported calls with non-zero symptoms. Items are ordered by overall frequency of reporting. $(\triangle)$ ). Females, baseline values; $(\boldsymbol{\Lambda})$, females, treatment values; $(\bigcirc)$, males, baseline values; $(\bullet)$, males, treatment values; Obstip, being disturbed by constipation; Borbor, borborygmi; DiaUrg, being disturbed by urgent need to defecate; DiaFreq, being disturbed by frequent bowel movements during last $3 \mathrm{~d}$. For further details of the questionnaire items of the automated telemedical data-capturing system, see Methods.

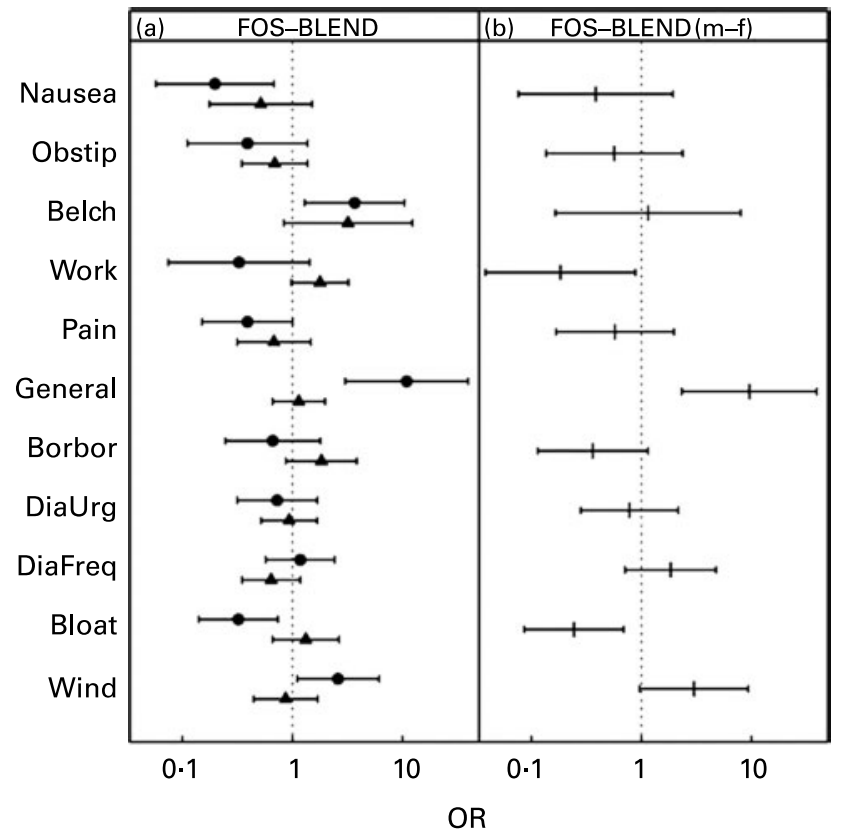

Fig. 2. (a) Symptom score OR on a logarithmic scale of once-daily ingestion for 5 weeks of $10 \mathrm{~g}$ food-grade fructo-oligosaccharide (FOS; Raftilose ${ }^{\circledR}$ P95) $v$. once-daily ingestion for 5 weeks of a $10 \mathrm{~g}$ prebiotic mixture of food-grade FOS and acacia gum $(1: 1$; BLEND) for females $(\boldsymbol{\Lambda})$ and males $(\bullet)$, with $95 \%$ $\mathrm{Cl}$ represented by horizontal bars. (b) Between-sexes FOS $v$. BLEND difference from interaction term in generalised linear mixed model. The dotted vertical line at the OR of 1 corresponds to 'no difference in reported frequency'. m, Males; $f$, females; Obstip, being disturbed by constipation; Borbor, borborygmi; DiaUrg, being disturbed by urgent need to defecate; DiaFreq, being disturbed by frequent bowel movements during last $3 \mathrm{~d}$. For further details of the questionnaire items of the automated telemedical data-capturing system, see Methods.

$(P=0.002)$. To check whether this was a statistical artifact of the GLMM method ${ }^{(23)}$, a Bayesian estimate ${ }^{(22)}$ was computed for this item giving an even larger OR for the sex difference of 27 (95\% CI 4.3, 121; $P$ values commonly not given for Bayesian methods).

The higher incidence of 'belching' under FOS ( $P=0.09$ for females; $P=0.01$ for males) had the same tendency for both sexes. 'Bloating' under FOS and BLEND was expressed differently for sex, as computed from the interaction term in the GLMM (Fig. 2 (b), and last column in Table 1; $P=0.008)$ with an OR of $1.3(=$ higher under FOS $)$ for females and $0.3(=$ lower under FOS $)$ for males. Most symptom scores increased during the treatment intervals and the run-in period (Fig. 3).

\section{Fructo-oligosaccharide and prebiotic mixture of fructo- oligosaccharide and acacia gum v. run-in}

The response scores for 'bloating' and 'wind' were significantly higher during FOS and BLEND treatment as compared with the pooled run-in period $\left(P<10^{-10}\right.$ for both items and sexes). Occurrence of 'borborygmi' was significantly increased in both phases $\left(P<10^{-5}\right)$. 'General' wellbeing under FOS was reduced compared with run-in (OR 5.4; $P<10^{-7}$ ), but less under BLEND (OR 2.9; $P=0.001$ ), an effect that was overlaid by the extreme sex differences for this item. 'Nausea' and 'reflux' were almost never reported. 
Table 1. Fructo-oligosaccharide (FOS) $v$. prebiotic mixture of FOS and acacia gum for primary endpoint questionnaire items, by sex* (Odds ratios)

\begin{tabular}{|c|c|c|c|c|c|c|}
\hline \multirow[b]{2}{*}{ Item } & \multicolumn{2}{|c|}{ Females } & \multicolumn{2}{|c|}{ Males } & \multicolumn{2}{|c|}{$\begin{array}{l}\text { Males- } \\
\text { females }\end{array}$} \\
\hline & OR† & $P$ & OR† & $P$ & OR† & $P$ \\
\hline Wind & 0.9 & 0.7 & $2 \cdot 6$ & 0.03 & $3 \cdot 0$ & 0.06 \\
\hline Bloat & 1.3 & 0.4 & 0.3 & 0.008 & 0.2 & 0.008 \\
\hline DiaFreq & 0.6 & 0.2 & $1 \cdot 2$ & 0.7 & 1.8 & 0.2 \\
\hline DiaUrg & 0.9 & 0.8 & 0.7 & 0.5 & $0 \cdot 8$ & 0.6 \\
\hline Borborygmi & 1.8 & $0 \cdot 1$ & 0.7 & 0.4 & 0.4 & 0.08 \\
\hline General & $1 \cdot 1$ & 0.6 & $10 \cdot 9$ & $3 \times 10^{-4}$ & $9 \cdot 6$ & 0.002 \\
\hline Pain & 0.7 & 0.3 & 0.4 & 0.05 & 0.6 & 0.4 \\
\hline Work & 1.8 & 0.06 & 0.3 & 0.1 & $0 \cdot 2$ & 0.03 \\
\hline Belch & 3.2 & 0.09 & 3.7 & 0.01 & $1 \cdot 2$ & 0.9 \\
\hline Obstip & 0.7 & 0.3 & 0.4 & 0.1 & 0.6 & 0.4 \\
\hline Nausea & 0.5 & 0.2 & 0.2 & 0.01 & 0.4 & 0.2 \\
\hline
\end{tabular}

* For abbreviations of the questionnaire items of the automated telemedical datacapturing system, see Methods.

†OR $>1$ indicate more frequent symptoms under FOS. The $P$ values test against the null hypothesis of an $\mathrm{OR}=1.0$; no correction for multiple testing has been applied.

During run-in only $14 \%$ of all responses to the questionnaire were larger than 0 indicating slight (in most of the cases), moderate, or strong effects. Of the responses under FOS and BLEND, $30 \%$ were larger than 0 .

\section{Determinants of gut comfort}

Irrespective of the treatments, in both sexes changes in gut symptoms affected 'general' wellbeing more strongly $\left(R^{2}>0.35\right)$ than wellbeing during 'work' $\left(R^{2}>0.20\right.$; Fig. 4). In men, 'borborygmi' were the strongest determinant of gut comfort $\left(R^{2}>0 \cdot 26\right)$. Stool-related symptoms were secondary for males. In priority sequence, 'bloating', 'wind' and 'stool frequency' were main determinants of wellbeing in women. 'Belching', which was significantly higher under FOS, is a major determinant for general wellbeing, but is not a relevant factor influencing work.

\section{Rectal barostat}

No significant effects of treatment type on rectal compliance and asymptotic compliance were detected. Threshold pressures and rectal sensations (urgency, wind, discomfort and pain) were also similar for all treatments during phasic distension (Table 2). In the GLMM analysis of dichotomised sensory responses the scores for urgency were higher under FOS compared with BLEND (OR 2.2; $P=0 \cdot 01$ ), with discomfort and pain showing the same tendency (Fig. 5).

\section{Stool analysis}

No differences in faecal bifidobacteria concentration and the number of total bacteria as well as bifidobacteria:total bacteria ratio were observed between FOS and BLEND (bifidobacteria: 9.8 (SD 0.3) v. 9.7 (SD 0.4) log colony-forming units/g; total bacteria: 10.4 (SD 0.3) v. 10.4 (SD 0.3) $\log$ colony-forming units/g).

\section{Discussion}

The main results of the present study were: (i) evidence to suggest that FOS generates slightly more abdominal side effects than acacia gum; (ii) indication of sex differences of determinants in gut wellbeing; (iii) confirmation that selfobservation induces a sensitisation or higher level of alertness to gut signals; (iv) that patient monitoring using an automated telemedical data-capturing system assures excellent questionnaire response rates.

The differences between scores for FOS and BLEND were small, i.e. smaller than those between both product periods and the run-in period; only belching was significantly stronger under FOS in both sexes. It remains to be elucidated whether the daily $10 \mathrm{~g}$ supplement was simply below the threshold for the induction of more pronounced effects, and to what extent sexes differ in their response to fibre supplementation.

Sex differences in transit time, faecal weight and biochemistry and in dietary fibre intake have been reported ${ }^{(24)}$ and either of these differences may explain the weaker sex-specific responses. The results of the present study support the occurrence of sex differences in response to prebiotic supplementation as has been observed in studies describing sex differences in response to dietary fibre supplements ${ }^{(25-27)}$. These effects surpass a purely symptomatic response: combined data from two large intervention trials ${ }^{(28)}$ revealed that dietary fibre both as a supplement or in a high-fibre diets protect men but not women against colorectal adenoma recurrence ${ }^{(26,28)}$. Sex differences in brain responses to rectal distention have also been reported, suggesting different neural processing or behavioural changes to the perception of gut stimuli ${ }^{(29-31)}$. Not surprisingly, in the present study sexes differed also in their perception of behavioural changes induced by ingestion of dietary prebiotics. These sex differences need to be taken in account when targeting populations of both sexes with dietary fibre supplements, as has already been done for recommended daily fibre intake. Further experiments are needed to confirm and characterise the sex differences in response to fibre ingestion. The inclusion of larger doses of fibre supplements would certainly answer the questions about threshold doses in both sexes, and could also confirm sex differences.

Both products significantly enhanced scores for bloating and wind. 'Borborygmi' were significantly enhanced under treatment with FOS. Moreover, there was a tendency for enhanced scores for belching and 'general' impairment under FOS only. Only $30 \%$ of all responses to the questionnaire during FOS or BLEND were larger than zero, suggesting that the supplemented dose had generally small effects on abdominal symptoms. Nonetheless, both FOS and BLEND showed significant effects as compared with the run-in phase with maltodextrin. It remains unclear whether carrying out the run-in period in an unblinded way may have contributed to the size of the effects between FOS or BLEND and runin. The increase in some of the symptoms during the treatment periods was rather surprising. In fact, adaptation to the $10 \mathrm{~g}$ supplement was to be expected; however, symptoms generally tended to increase over time (Fig. 3). The increase was also observed during the run-in (maltodextrin) periods, suggesting that it is rather a higher level of alertness in self-observance that leads to the 'sensitisation' than the product itself. 

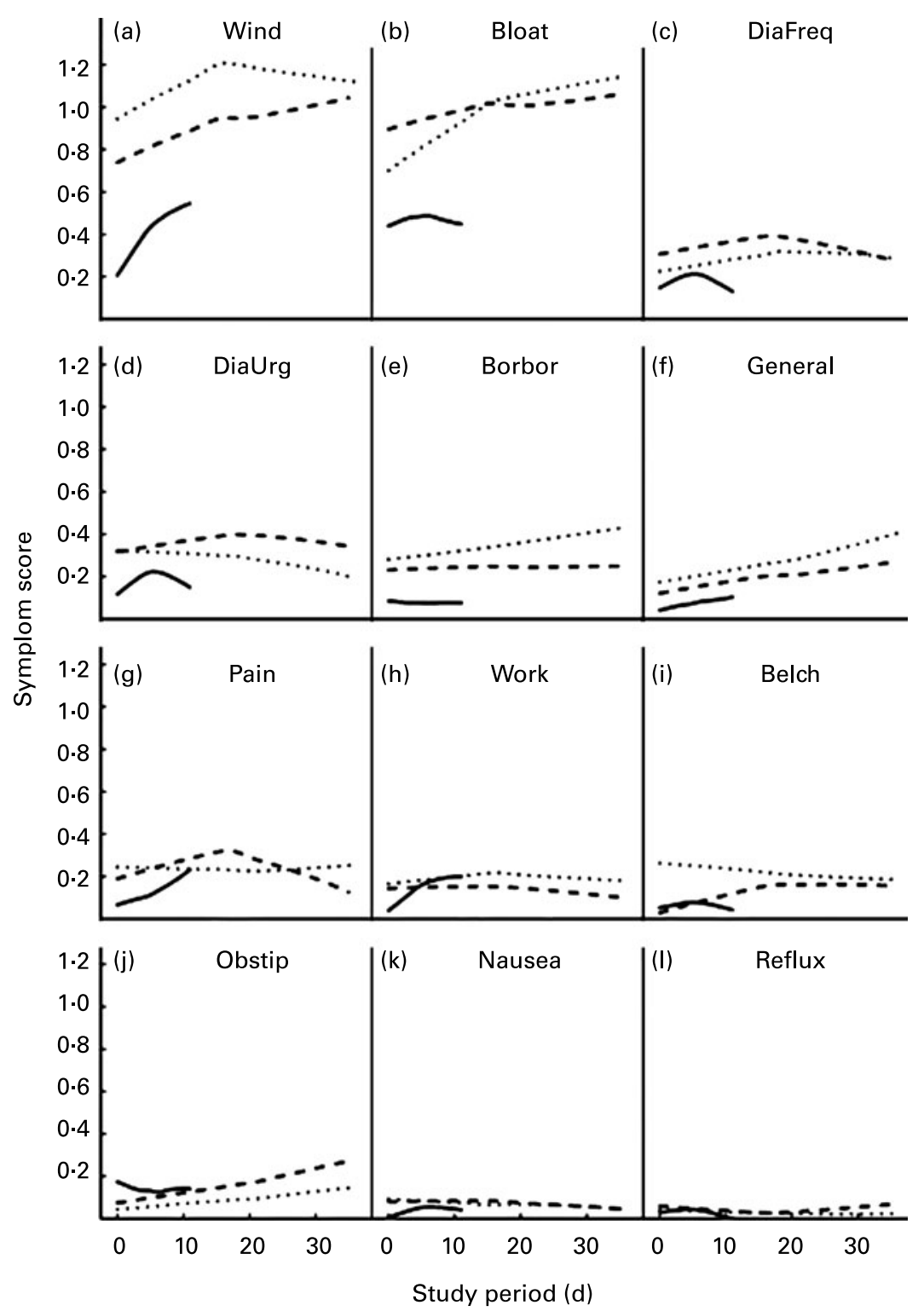

Fig. 3. Smoothed time series of symptom scores by treatment. (-), Pooled score results of the two 2 -week baseline phases with $10 \mathrm{~g}$ maltodextrin once daily (run-in); (...), score results of the 5-week ingestion period of $10 \mathrm{~g}$ food-grade fructo-oligosaccharide (FOS; Raftilose ${ }^{\circledR}$ P95) once daily; (--), score results of the 5 -week ingestion period of a $10 \mathrm{~g}$ mixture (1:1) of FOS and acacia gum once daily (BLEND). The symptoms are (a) wind, (b) bloating, (c) being disturbed by frequent bowel movements during last $3 \mathrm{~d}$, (d) being disturbed by urgent need to defecate, (e) borborygmi, (f) general wellbeing, ( $\mathrm{g}$ ) pain, (h) reducing daily activity (work), (i) belching, (j) being disturbed by constipation, (k) nausea, (l) reflux. For further details of the questionnaire items of the automated telemedical data-capturing system, see Methods.

The sensory score for 'urgency' during the barostat test was significantly higher under FOS than under BLEND, with 'discomfort' and 'pain' having the same tendency. The differences between FOS and BLEND point in the same direction as the questionnaire results. An increase in urgency in the questionnaire, with no additional stimulus applied, was not observed under FOS.

To evaluate further differences between FOS and BLEND, concentrations of bifidobacteria as indicators of gut health were quantified. There were no significant differences in gut bifidobacteria concentration or total bacterial content between FOS and BLEND, probably due to the low dose of prebiotic supplemented in the present study.
Possibly, the replacement of a large proportion of the fibres ingested with the regular diet by FOS or BLEND would have helped to reveal differences between the supplements as well as between the run-in periods and the supplements. This had not been addressed in the present study to avoid increasing the cumbersome stool collections to a total of four collections.

In general, prebiotics are safe and mainly well-tolerated food supplements with only slight differences as observed between the products FOS and BLEND. However, BLEND did show a slightly more favourable side-effect profile in comparison with FOS, supporting the concept of slower fermentation in the genesis of abdominal symptoms. Further 


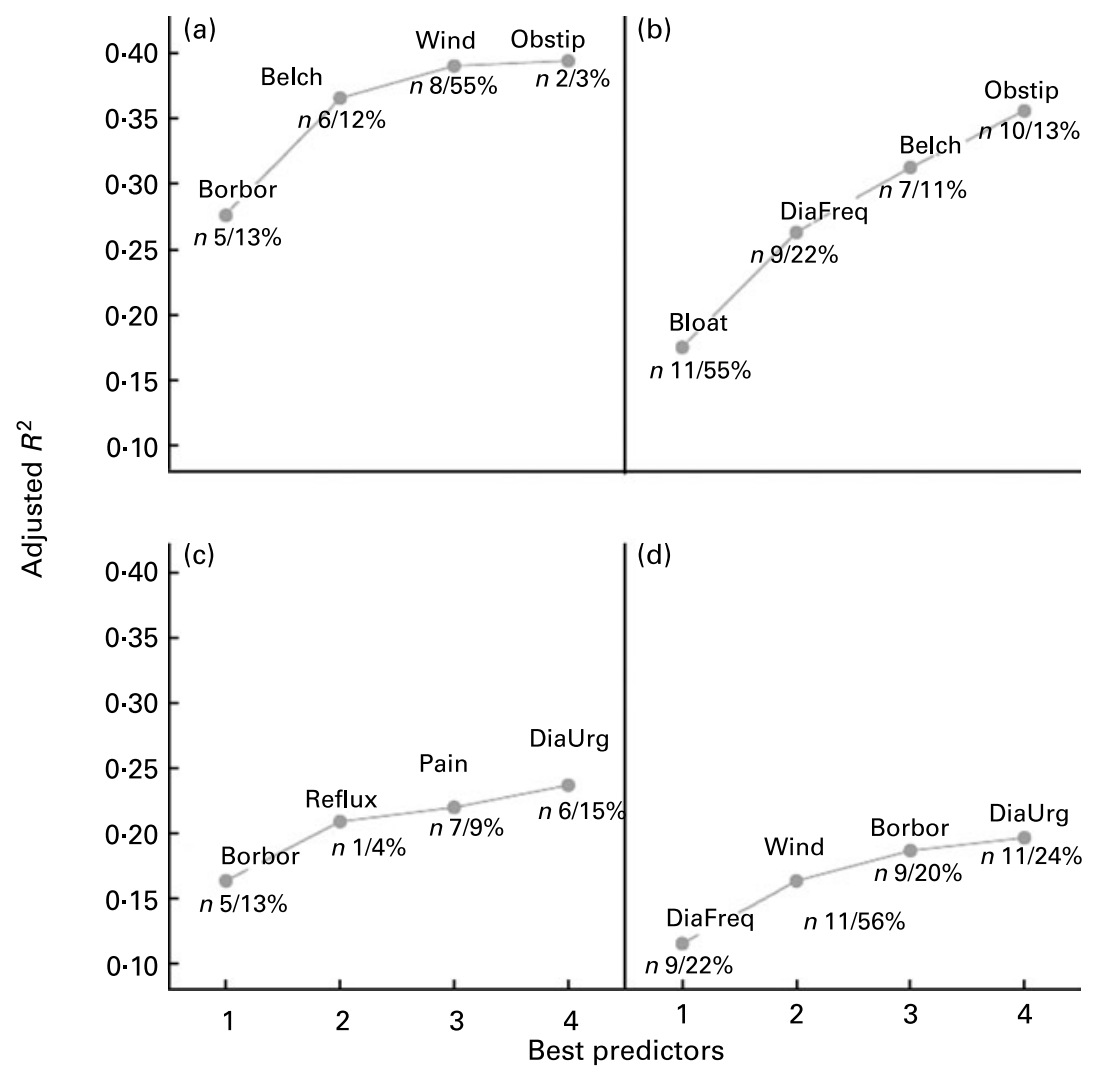

Fig. 4. Main determinants of intestinal symptoms for items 'General' (reduced general wellbeing) (a and b) and 'Work' (influence at work) (c and d) for males ( $a$ and $c$ ) and females (b and d). Initially all ten other questionnaire item scores were used to predict 'General' and 'Work' score respectively, and an Akaike information criterion-controlled subsets regression reduced predictors to the four most dominant ones. The vertical scale shows adjusted squared correlation coefficient; for example, for $n 5 / 13 \%$, five of the eight male subjects included in the study reported non-zero symptom score values, and overall $13 \%$ of the responses were $>0$. borbor, Borborygmi; obstip, being disturbed by constipation; DiaFreq, being disturbed by frequent bowel movements during last $3 \mathrm{~d}$; DiaUrg, being disturbed by urgent need to defecate. For further details of the questionnaire items of the automated telemedical data-capturing system, see Methods.

Table 2. Rectal function and sensations after a 5-week period of fructo-oligosaccharide (FOS) or prebiotic mixture of FOS and acacia gum (BLEND) ingestion as assessed by barostat*

(Medians and interquartile ranges (IQR) for twenty subjects)

\begin{tabular}{|c|c|c|c|c|}
\hline \multirow[b]{2}{*}{ Rectal barostat } & \multicolumn{2}{|c|}{ FOSt } & \multicolumn{2}{|c|}{ BLEND $\ddagger$} \\
\hline & Median & IQR & Median & IQR \\
\hline Rectal pressure $(\mathrm{mmHg})$ & $3 \cdot 7$ & $1.9-4.0$ & $3 \cdot 4$ & $2 \cdot 7-4 \cdot 8$ \\
\hline Rectal compliance $(\mathrm{ml} / \mathrm{mmHg})$ & $16 \cdot 1$ & $12 \cdot 6-20 \cdot 8$ & $15 \cdot 7$ & $13 \cdot 7-25 \cdot 8$ \\
\hline Asymptotic compliance $(\mathrm{ml} / \mathrm{mmHg})$ & 4.93 & $4 \cdot 01-6 \cdot 35$ & $5 \cdot 43$ & $4.97-6.43$ \\
\hline \multicolumn{5}{|l|}{ Threshold pressures } \\
\hline Vague $(\mathrm{mmHg})$ & $6 \cdot 35$ & $3 \cdot 98-8 \cdot 35$ & $7 \cdot 29$ & $5 \cdot 66-9 \cdot 88$ \\
\hline Pain $(\mathrm{mmHg})$ & $35 \cdot 9$ & $26 \cdot 1-41 \cdot 9$ & $39 \cdot 9$ & $25 \cdot 7-42 \cdot 9$ \\
\hline \multicolumn{5}{|l|}{ Rectal sensation at $36 \mathrm{mmHg}$} \\
\hline VAS urgency (mm) & 74 & $47-92$ & 62 & $46-84$ \\
\hline VAS wind (mm) & 14 & $0-53$ & 31 & $5-47$ \\
\hline VAS discomfort (mm) & 83 & $48-93$ & 66 & $48-97$ \\
\hline VAS pain $(\mathrm{mm})$ & 55 & $19-76$ & 38 & $10-78$ \\
\hline
\end{tabular}

VAS, visual analogue scale.

* No significant differences in sensory scores were found using a mixed model analysis, and there were no differences in rectal functional parameters and threshold pressures by exact Wilcoxon matched pair tests.

†Functional parameters after a 5-week ingestion period of $10 \mathrm{~g}$ food-grade Raftilose ${ }^{\circledR} \mathrm{P} 95$ once daily

† Functional parameters after a 5-week ingestion period of a $10 \mathrm{~g}$ mixture $(1: 1)$ of FOS and acacia gum once daily. 


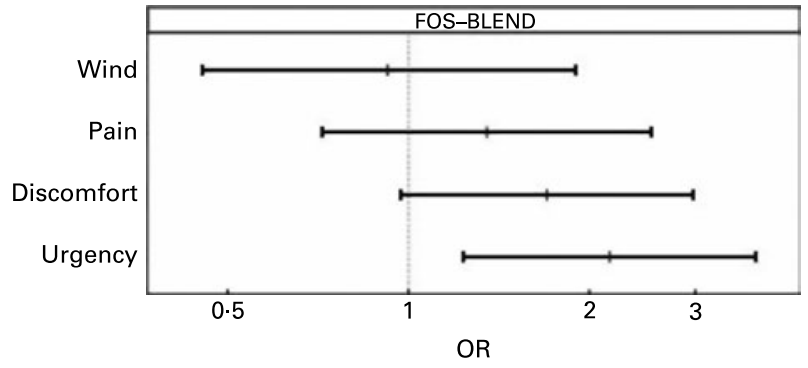

Fig. 5. Generalised linear mixed model estimates of OR and $95 \% \mathrm{Cl}$ (represented by horizontal bars) of rectal sensations during phasic rectal barostat distensions. Values above 1 indicate that higher symptoms were reported under fructo-oligosaccharide (FOS) compared with the prebiotic mixture of FOS and acacia gum (BLEND).

experiments are needed to confirm and characterise the sex differences in response to fibre ingestion. The inclusion of larger doses of fibre supplements would clarify the role of sex-specific threshold doses.

\section{Acknowledgements}

All authors contributed equally for intellectual input and writing of the manuscript. D. M. and O. G. performed the statistical analysis. M. G., F. R. and K. O. are employed by the Nestlé Research Centre. All authors declare no conflict of interest. The work was funded by a grant from the Nestlé Research Centre.

Marianna Giarrè passed away before submission of the manuscript. Because of her friendly, warm and constructive attitude it was always a pleasure to interact with her.

The excellent technical assistance of Dr U. Sahrbacher and Bernadette Stutz is greatly acknowledged.

\section{References}

1. Cummings JH, Gibson GR \& Macfarlane GT (1989) Quantitative estimates of fermentation in the hind gut of man. Acta Vet Scand Suppl 86, 76-82.

2. Gibson GR \& Roberfroid MB (1995) Dietary modulation of the human colonic microbiota: introducing the concept of prebiotics. J Nutr 125, 1401-1412.

3. Gibson GR (1998) Dietary modulation of the human gut microflora using prebiotics. Br J Nutr 80, S209-S212.

4. Cummings JH \& Macfarlane GT (2002) Gastrointestinal effects of prebiotics. Br J Nutr 87, Suppl. 2, S145-S151.

5. Brunser O, Figueroa G, Gotteland M, Haschke-Becher E, Magliola C, Rochat F, Cruchet S, Palframan R, Gibson G, Chauffard F \& Haschke F (2006) Effects of probiotic or prebiotic supplemented milk formulas on fecal microbiota composition of infants. Asia Pac J Clin Nutr 15, 368-376.

6. Ito M, Deguchi $\mathrm{Y}$, Matsumoto K, Kimura M, Onodera N \& Yajima $T$ (1993) Influence of galactooligosaccharides on the human fecal microflora. J Nutr Sci Vitaminol (Tokyo) 39, 635-640.

7. Gibson GR \& Wang X (1994) Regulatory effects of bifidobacteria on the growth of other colonic bacteria. J Appl Bacteriol 77, 412-420.

8. Coudray C, Bellanger J, Castiglia-Delavaud C, Remesy C, Vermorel M \& Rayssignuier Y (1997) Effect of soluble or partly soluble dietary fibres supplementation on absorption and balance of calcium, magnesium, iron and zinc in healthy young men. Eur J Clin Nutr 51, 375-380.

9. Roberfroid MB (2000) Prebiotics and probiotics: are they functional foods? Am J Clin Nutr 71, 1682S-1690S.

10. Bouhnik Y, Coffin B \& Rambaud JC (1996) Chronic idiopathic constipation in adults. Pathogenesis and treatment (article in French). Ann Gastroenterol Hepatol (Paris) 32, $89-96$.

11. Reddy BS (1998) Prevention of colon cancer by pre- and probiotics: evidence from laboratory studies. Br J Nutr 80, S219-S223.

12. Jackson KG, Taylor GR, Clohessy AM \& Williams CM (1999) The effect of the daily intake of inulin on fasting lipid, insulin and glucose concentrations in middle-aged men and women. Br J Nutr 82, 23-30.

13. Food Standards Australia New Zealand (2007) Australia New Zealand Food Standards Code, 1.2.8.18(1) Table. Barton, ACT, Australia: Food Standards Australia New Zealand.

14. Grimble G (1989) Fibre, fermentation, flora, and flatus. Gut 30, 6-13.

15. Marteau P \& Flourie B (2001) Tolerance to low-digestible carbohydrates: symptomatology and methods. Br J Nutr 85, Suppl. 1, S17-S21.

16. Rochat F, Baumgartner M, Jann A, Rochat I \& Ballevre O (2001) Synergistic effect of prebiotics on human intestinal microflora. In Congress of Probiotic Medicine, Annaheim, CA, July 2001.

17. Cherbut C, Michel C, Raison V, Kravtchenko T \& Severine M (2003) Accacia gum is a bifidogenic dietary fibre with high digestive tolerance in healthy humans. Microbial Ecol Health Dis 15, 43-50.

18. Thumshirn M, Coulie B, Camilleri M, Zinsmeister AR, Burton DD \& Van Dyke C (2000) Effects of alosetron on gastrointestinal transit time and rectal sensation in patients with irritable bowel syndrome. Aliment Pharmacol Ther 14, 869-878.

19. Fox M, Stutz B, Menne D, Fried M, Schwizer W \& Thumshirn $M$ (2005) The effects of loperamide on continence problems and anorectal function in obese subjects taking orlistat. Dig Dis Sci 50, $1576-1583$.

20. Pinheiro J \& Bates D (2000) Mixed-effects Models in S and $S$-PLUS. New York, Berlin and Heidelberg: Springer.

21. Venables WN \& Ripley BD (2002) Modern Applied Statistics with $\{S\}$. New York: Springer.

22. Jara A (2007) Bayesian nonparametric and semiparametric analysis http://cran.r-project.org/web/packages/DPpackage/index. html

23. Hauck WW Jr \& Donner A (1977) Wald's test as applied to hypotheses in logit analysis. $J$ Am Stat Assoc 72, $851-853$

24. Lampe JW, Fredstrom SB, Slavin JL \& Potter JD (1993) Sex differences in colonic function: a randomised trial. Gut 34, 531-536.

25. Martinez ME, McPherson RS, Annegers JF \& Levin B (1996) Association of diet and colorectal adenomatous polyps: dietary fiber, calcium, and total fat. Epidemiology 7, 264-268.

26. Fuchs CS, Giovannucci EL, Colditz GA, Hunter DJ, Stampfer MJ, Rosner B, Speizer FE \& Willett WC (1999) Dietary fiber and the risk of colorectal cancer and adenoma in women. $N$ Engl $\mathrm{J}$ Med 340, 169-176.

27. Mathew A, Peters U, Chatterjee N, Kulldorff M \& Sinha R (2004) Fat, fiber, fruits, vegetables, and risk of colorectal adenomas. Int J Cancer 108, 287-292.

28. Jacobs ET, Lanza E, Alberts DS, Hsu CH, Jiang R, Schatzkin A, Thompson PA \& Martinez ME (2006) Fiber, sex, and colorectal adenoma: results of a pooled analysis. Am J Clin Nutr 83, 343-349. 
29. Berman S, Munakata J, Naliboff BD, Chang L, Mandelkern M, Silverman D, Kovalik E \& Mayer EA (2000) Gender differences in regional brain response to visceral pressure in IBS patients. Eur J Pain 4, 157-172.

30. Kern MK, Jaradeh S, Arndorfer RC, Jesmanowicz A, Hyde J \& Shaker R (2001) Gender differences in cortical representation of rectal distension in healthy humans. Am J Physiol Gastrointest Liver Physiol 281, G1512-G1523.

31. Lawal A, Kern M, Sanjeevi A, Hofmann C \& Shaker R (2005) Cingulate cortex: a closer look at its gut-related functional topography. Am J Physiol Gastrointest Liver Physiol 289, G722-G730. 